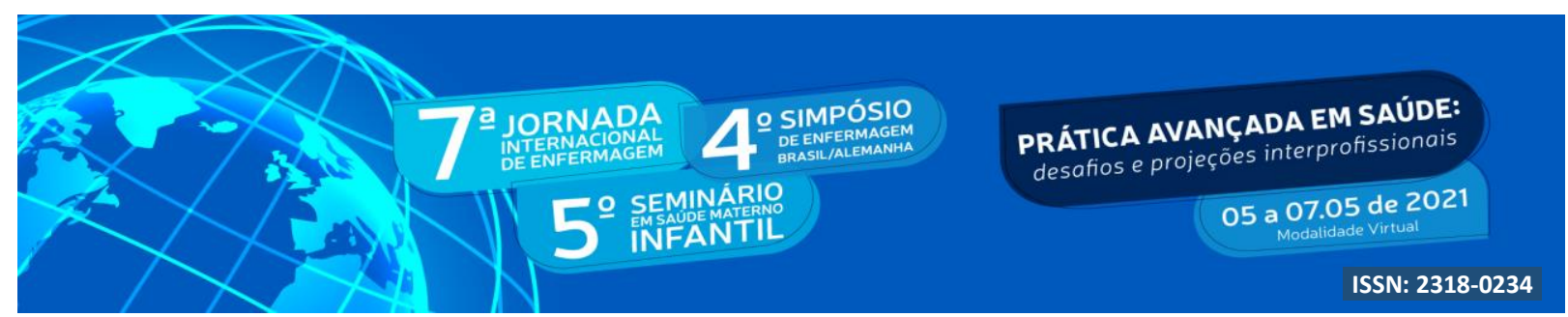

DOI: http://doi.org/10.48195/jie2021-052

\title{
PROCESSO DE ENCAMINHAMENTO DE GESTANTES PORTADORAS DE HIV EM SANTA MARIA ${ }^{1}$
}

\author{
Natalia Cassol Bolzan²; Giovana Batistella de Melo ${ }^{3}$; Manuela Dalenogare ${ }^{4}$; Mara \\ Regina Caino Teixeira Marchiori ${ }^{5}$
}

\begin{abstract}
RESUMO
Objetiva-se relatar a experiência de acadêmicas de enfermagem na pesquisa e construção de um fluxograma sobre o processo de encaminhamento de gestantes com HIV nas Redes de Atenção à Saúde no município de Santa Maria/RS. O presente estudo é um relato de experiência realizado como atividade de ensino na disciplina de Processo de Trabalho em Saúde, no segundo semestre de 2020. Este fluxograma irá auxiliar os profissionais da saúde, tanto da atenção básica quanto dos hospitais, no encaminhamento e o como intervir em cada situação frente a gestantes com HIV, como também ser esclarecedor para os usuários, visto que ao observarem o fluxograma, irão visualizar qual serviço devem procurar. Conclui-se que é extremamente importante a realização do pré-natal e a adesão ao tratamento em pacientes gestantes, pois quando ambos são feitos corretamente e com um acompanhamento profissional, os riscos de complicações e de transmissão vertical diminuem.
\end{abstract}

Palavras-chave: Gestação; Pré-natal; Rede de Atenção à Saúde.

\begin{abstract}
This study aims to report the experience of nursing students in researching and building a flowchart on the referral process of pregnant women with HIV in the Health Care Networks in the city of Santa Maria/RS. This study is an experience report carried out as a teaching activity in the discipline of Work Process in Health, in the second semester of 2020. This flowchart will help health professionals, both in primary care and hospitals, in the referral and how to intervene in each situation facing pregnant women with HIV, as well as being enlightening for users, since by observing the flowchart, they will see which service they should seek. We conclude that it is extremely important to perform prenatal care and adherence to treatment in pregnant patients, because when both are done correctly and with a professional follow-up, the risks of complications and vertical transmission decrease.
\end{abstract}

Key Words: Health Care Network; Pregnancy; Prenatal.

\footnotetext{
${ }^{1}$ Relato de experiência

${ }^{2}$ Estudante do Curso de Enfermagem. Universidade Franciscana (UFN). E-mail: natalia_cassol@ hotmail.com

${ }^{3}$ Estudante do Curso de Enfermagem. Universidade Franciscana (UFN). E-mail: giovanamello20@ outlook.com

${ }^{4}$ Estudante do Curso de Enfermagem. Universidade Franciscana (UFN). E-mail: dalenogaremanu@ hotmail.com

${ }^{5}$ Orientadora. Professora do Curso de Enfermagem. Universidade Franciscana (UFN). E-mail:

mara.marc@hotmail.com 


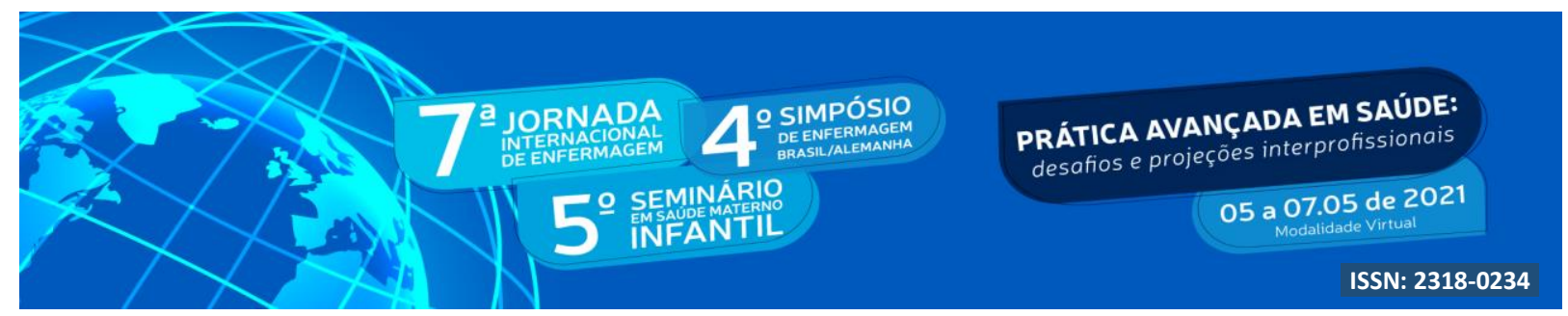

\section{INTRODUÇÃ̃O}

A gestação é um processo fisiológico na vida da mulher que envolverá mudanças físicas, emocionais, sociais e psicológicas. Ao detectá-la, deve-se iniciar imediatamente o prénatal. Os atendimentos começam pela principal porta de entrada do Sistema Único de Saúde (SUS), a Atenção Básica (AB), ou seja, nas Unidades Básicas de Saúde (UBS) e Estratégias de Saúde da Família (ESF) (BRASIL, 2013).

A $\mathrm{AB}$ deve ser a porta de entrada preferencial da gestante no SUS, bem como o ponto de atenção estratégico para atender suas necessidades e realizar um acompanhamento continuado. Esta, é caracterizada por ser um conjunto de ações no âmbito coletivo e individual, executadas por equipes multiprofissionais, visando à proteção e promoção da saúde, reabilitação, diagnóstico, manutenção e tratamento à saúde e prevenção de agravos (BRASIL, 2012).

O pré-natal é realizado na $\mathrm{AB}$ e tem como objetivo garantir o desenvolvimento da gestação, possibilitando o nascimento saudável de um recém-nascido (RN), sem complicações para a saúde materna, bem como abordar aspectos psicossociais e atividades de prevenção e educativas (BRASIL, 2013).

O número ideal de consultas é igual ou superior a seis, sendo iniciado no primeiro trimestre gestacional. Devem ser mensais até a $28^{\mathrm{a}}$ semana, quinzenais entre 28 e 36 semanas e semanais após esse período. Não há alta do pré-natal. O enfermeiro é o profissional que realiza tais consultas, o qual têm como principais competências realizar testes rápidos nas gestantes, solicitar exames complementares e identificar quais mulheres possuem algum risco, bem como identificar se a gestação é de alto risco e encaminhar para consultas médicas (BRASIL, 2013).

Também é uma etapa que poderá ocasionar riscos maternos e fetais, havendo um quantitativo de gestantes que por fatores particulares, têm maior probabilidade de evoluir de forma contrária do que se espera, sendo considerada "gestação de alto risco". Dessa maneira, deve-se encaminhar diretamente ao serviço de referência do município (BRASIL, 2013).

Objetivando diminuir a morbimortalidade materno-infantil e aumentar o acesso de resolutividade a esse público, se faz necessária a identificação dos fatores de risco da gestação 


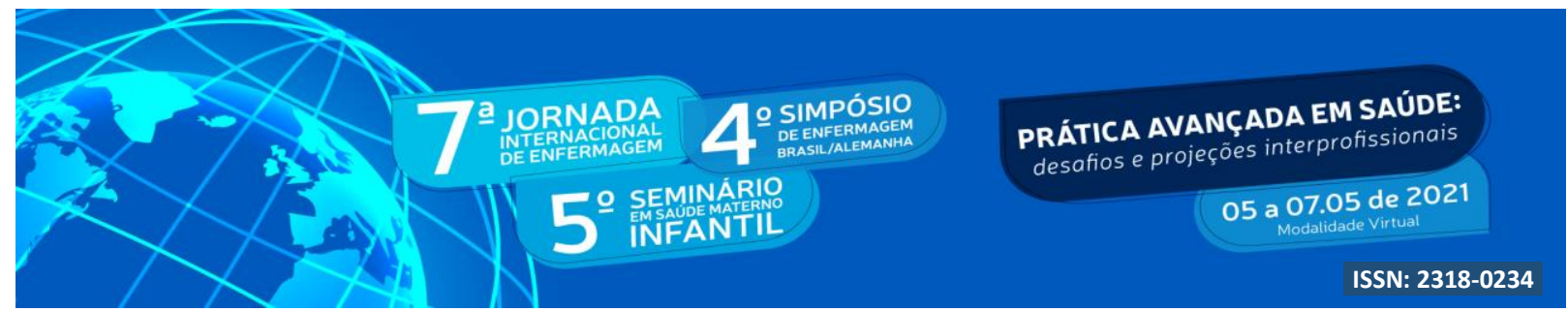

de forma precoce. Para assim, o acolhimento com a classificação de risco auxiliar na agilidade da assistência e identificação da necessidade de intervenções (BRASIL, 2013).

A adesão das gestantes ao pré-natal está relacionada à qualidade dos cuidados prestados pelos profissionais de saúde. Entende-se que para essa adesão ocorrer é necessário que o profissional esteja preparado para atuar nesse serviço, proporcionando acolhimento, humanização, levando em consideração seus aspectos socioculturais, ouvindo-as e proporcionando um ambiente confortante para a gestante e sua família (VIELLAS et al., 2014).

Dentre os fatores de risco que caracterizam uma gestação de alto risco, está a infecção pelo vírus HIV (imunodeficiência humana), causador da aids (imunodeficiência adquirida) (BRASIL, 2013). O HIV acomete o sistema imunológico no organismo, assim como, transmite a aids. O vírus é transmitido por meio de relações sexuais sem proteção com pessoas infectadas, pelo compartilhamento de objetos perfuro-cortantes, e de mães soropositivas sem tratamento para o bebê durante a gravidez, parto ou amamentação, chamando de transmissão vertical (BRASIL, 2017).

As gestantes diagnosticadas durante o pré natal, deverão fazer o tratamento antirretroviral ao longo da gestação e, caso necessário, também no parto. O tratamento irá prevenir a transmissão vertical para o bebê. Além de receber a medicação antirretroviral, a criança será acompanhada pelo serviço de saúde. A amamentação é uma prática não recomendada em casos de mães soropositivas, visto que poderá ocorrer a transmissão do vírus através do leite materno (BRASIL, 2020).

\section{OBJETIVO}

O presente artigo tem como objetivo descrever e relatar a experiência de acadêmicas de enfermagem na pesquisa e construção de um fluxograma sobre o processo de encaminhamento de gestantes com HIV nas Redes de Atenção à Saúde.

\section{METODOLOGIA}




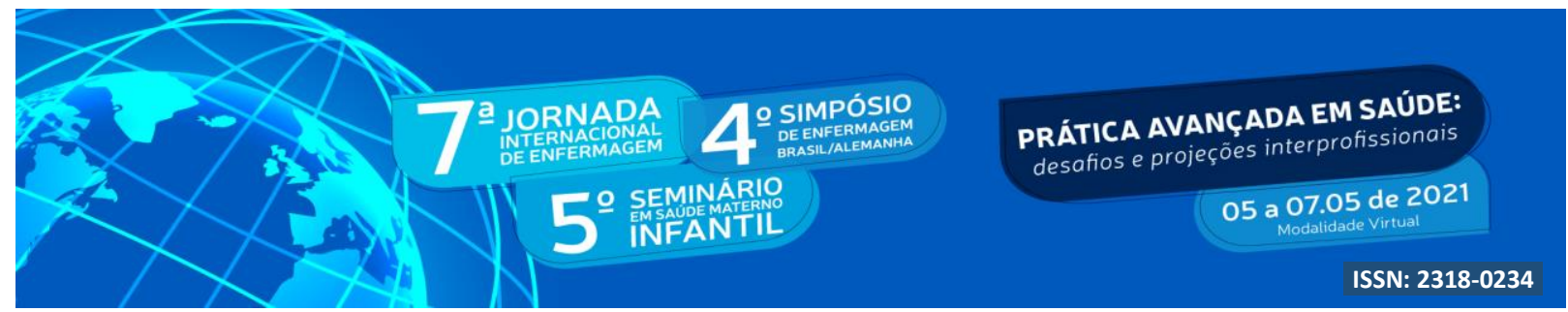

Trata-se de um estudo do tipo relato de experiência, descritivo de abordagem qualitativa, cujas vivências teórico-práticas foram realizadas como atividade de ensino na disciplina de Processo de Trabalho em Saúde, cursada ao longo do oitavo semestre do curso de Enfermagem, no segundo semestre do ano de 2020, sob a orientação da professora responsável pela disciplina.

O relato de experiência traduz-se em uma forma metodológica que possibilita descrever experiências vivenciadas, sendo de grande relevância para a formação dos futuros profissionais em saúde. Em seu meio acadêmico, contribui com a discussão, análise e proposição de estratégias para a melhoria do assunto abordado (GIL, 2007).

A construção deste fluxograma analisador deu-se em três momentos distintos. No primeiro momento os alunos foram divididos em grupos, onde cada grupo optou pelo tema que gostaria de abordar. Após a seleção dos temas e em segundo momento, as acadêmicas realizaram pesquisas bibliográficas para melhor apropriar-se do tema e entraram em contato com a responsável pela Política de Saúde da Mulher e da Criança e também com a Política de HIV/Aids, IST's e Hepatites Virais da Secretaria Municipal de Saúde (SMS) de Santa Maria/RS. E por último, realizou-se reuniões online com o grupo para o desenvolvimento e construção do fluxograma.

Salienta-se que as atividades foram realizadas em dias e horários fora dos períodos de aula e de maneira remota, visto que ainda persistia o momento pandêmico.

\section{RESULTADOS E DISCUSSÃO}

As acadêmicas de enfermagem realizaram um fluxograma sobre o processo de encaminhamento de gestantes com HIV baseado na realidade local do município de Santa Maria/RS. Esse fluxograma tem como intuito auxiliar os profissionais da saúde, tanto da AB quanto dos hospitais, no encaminhamento e como intervir em cada situação frente a gestantes com HIV, como também ser esclarecedor para os usuários, visto que ao observarem o fluxograma, irão visualizar qual serviço devem procurar.

Foi demonstrado desde a entrada da mulher na AB. Iniciou-se pelo acolhimento, pois todo o processo de cuidado parte dele por ser o primeiro atendimento dentro do serviço. Este, 


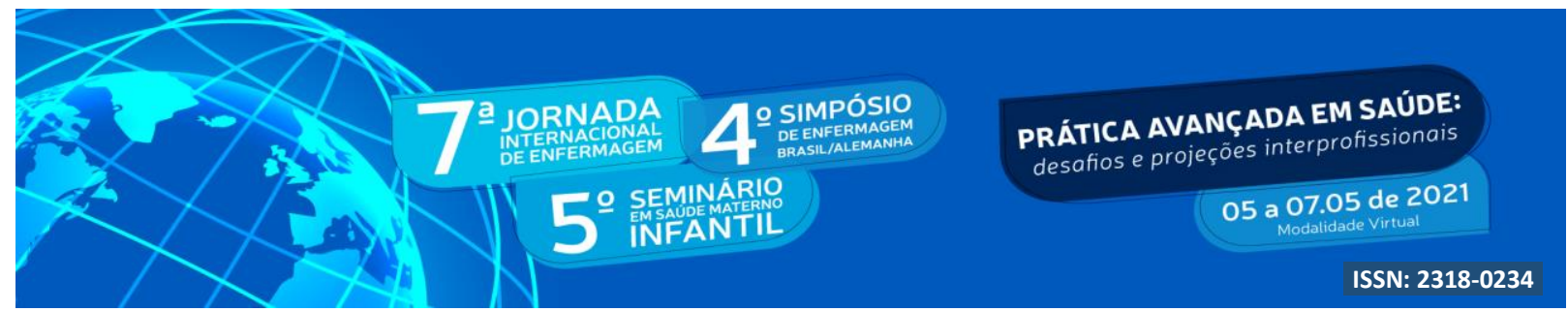

tem como objetivo aproximar o profissional da saúde com a usuária. Além disso, esta aproximação contribui ativamente no tratamento, que em diversas vezes se não efetivados com qualidade, os mesmos deixam de procurar assistência ao serviço e a não aderência ao tratamento. A partir do acolhimento é realizado os exames para detectar se a usuária está grávida, e se for positivo, deve-se iniciar imediatamente o pré-natal.

O acolhimento, de forma geral, pode ser compreendido como a maneira como o profissional de saúde recepciona a usuária desde a sua entrada no local de saúde. O profissional deve-se tornar responsável por ela, ouvir atentamente suas queixas, sofrimentos e preocupações, e simultaneamente, colaborar para amenizar ou curar suas necessidades de saúde ou doença, com intuito de garantir, juntamente a outros serviços, a continuidade da assistência (BRASIL, 2014).

No fluxograma, foi destacado que há tanto o pré-natal da gestante quanto o pré-natal do parceiro. O último, é pouco conhecido pela maioria da população, mas os profissionais devem ressaltar a importância da participação do parceiro durante o pré-natal, visto que interfere até mesmo em condições de Infecções Sexualmente Transmissíveis (IST).

$\mathrm{Na}$ anamnese, é necessário que o profissional saliente a necessidade de abordagem ao parceiro da gestante, aborde os hábitos de vida e práticas da mesma, identifique fatores que necessitam de intervenção e possíveis sinais e sintomas, reforce os métodos de prevenção e transmissão, destaque os efeitos do tratamento na qualidade de vida, dentre outras informações em saúde (BRASIL, 2010).

É realizado o exame físico e testes rápidos na gestante, os quais tem como intuito detecção precoce de alguma patologia e encaminhamento a outros serviços de saúde. No fluxograma, destaca-se que a gestante deve realizar testes rápidos de IST's, caso algum dê positivo, deve ser testado também no parceiro.

As gestantes com HIV devem realizar testes rápidos desde o começo da gravidez até o momento do parto, caso testem positivo os parceiros também necessitam realizar o teste. Dessa forma, o tratamento deve iniciar de imediato com antirretrovirais durante a gestação até o trabalho de parto (BRASIL, 2017).

Foi ilustrado no fluxograma como "diagnóstico de HIV reagente", a partir disso, o profissional de saúde deve realizar intervenções imediatas, como o aconselhamento à gestante 


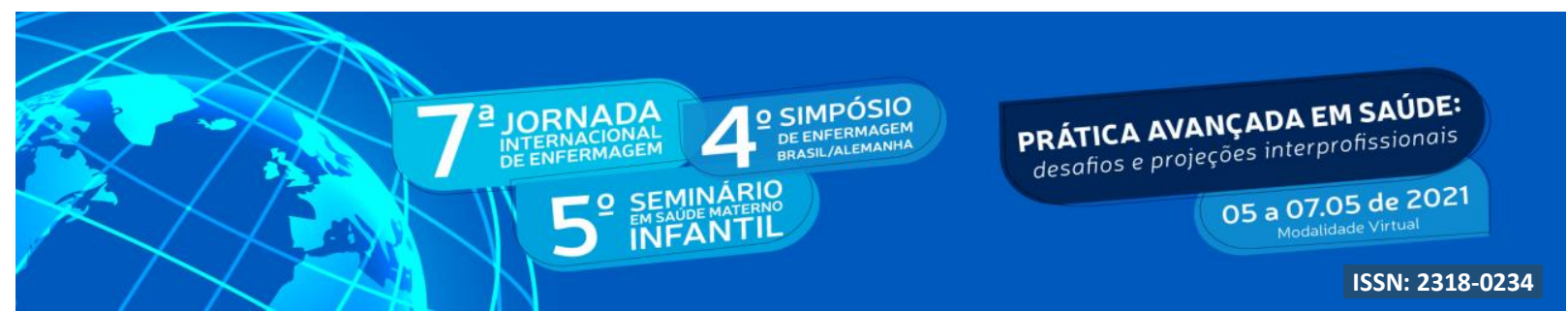

e seu parceiro, notificação, solicitação de determinados exames e encaminhamento ao prénatal de alto risco.

Receber o resultado positivo é difícil tanto para as gestantes, como para a equipe de saúde envolvida. Por isso, é preciso que os profissionais estejam capacitados para prestar assistência e amparo emocional, respeitando-a, acolhendo-a, ofertando informações à cerca da doença, possibilidades de tratamento e medidas de prevenção para evitar a transmissão vertical. Essas orientações e abordagens devem fazer sentido à gestante, ou seja, estarem de acordo com o grau de escolaridade e a condição emocional da mesma (BRASIL, 2010).

Na detecção do HIV, a notificação é obrigatória. As gestantes soropositivas devem ser encaminhadas para o pré-natal de alto risco ou Serviço de Atenção Especializada (SAE), conforme cada caso, devendo sempre manter um vínculo com a AB (BRASIL, 2019). A detecção precoce do vírus auxilia na adoção de medidas que irão diminuir o risco de transmissão vertical, ou seja, transmissão ao feto. Entre a população brasileira, estima-se que 0,4\% das gestantes sejam soropositivas para o vírus do HIV, o que se traduz em torno de 12.635 portadoras ao ano (BRASIL, 2010).

Conforme exposto no fluxograma, as gestantes que recebem o resultado positivo para HIV devem realizar alguns exames, quais são a contagem de linfócitos T-CD4 (LT-CD4+), Carga Viral do HIV (CV-HIV) e genotipagem. Devem também, ser encaminhadas ao prénatal de alto risco, de forma que os profissionais identifique-as via sistema.

A contagem de LT-CD4+ deve ser feita na primeira consulta do pré-natal e no mínimo a cada três meses para aquelas gestantes que iniciaram o tratamento. A CV-HIV serve para monitorar a gestante, avaliar o risco de transmissão vertical, auxiliar na avaliação da resposta a Terapia Antirretroviral (TARV) e contribui para o seguimento do tratamento e escolha da via de parto. Deve ser realizado no mínimo três exames durante o pré-natal, sendo na primeira consulta, para observar o nível de viremia, o segundo depois de duas a quatro semanas após iniciar a TARV e a partir de 34 semanas para instituir a via de parto (BRASIL, 2019).

No município de Santa Maria/RS, as gestantes que forem detectadas HIV positivo devem ser encaminhadas para um pré-natal de alto risco. Quando a carga viral for indetectável, pode realizar o tratamento na ESF de referência ou na Casa Treze de Maio, o qual é um SAE. Àquelas que detectaram o HIV no decorrer da gestação ou que aderiram ao 


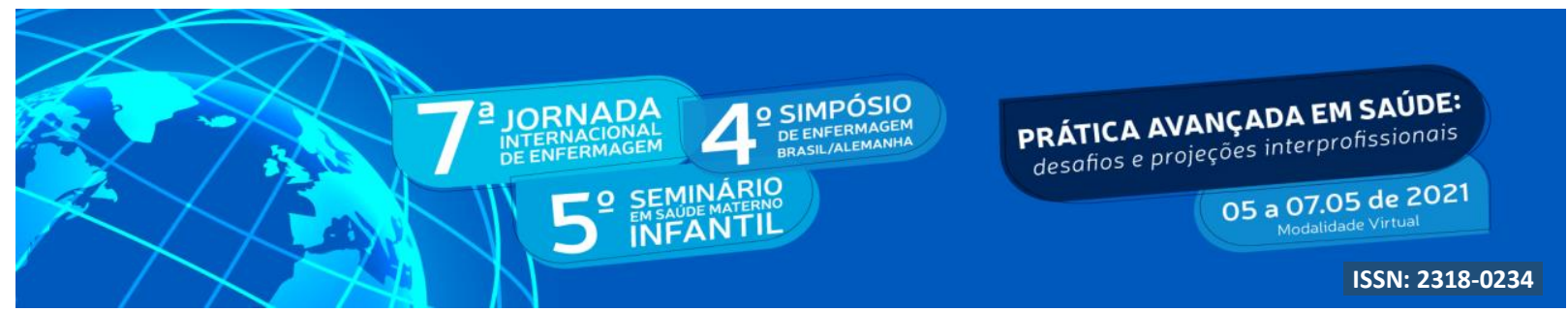

tratamento, devem ser direcionadas ao alto risco do Hospital Universitário de Santa Maria (HUSM). As gestantes podem optar por retirar os medicamentos da TARV na Casa Treze de Maio ou no HUSM.

Durante o pré-natal de gestantes reagentes para HIV, o profissional deve realizar: um acolhimento de qualidade, de maneira a coletar informações para um amplo entendimento sobre condições de vida que a mesma está inserida, identificar o nível de conhecimento da gestante referente a doença e o quão disposta está a seguir o tratamento. Após a primeira consulta de pré-natal, deve-se salientar a importância do tratamento e os riscos se não efetivar, e assim, encaminhar a gestante para o local de referência para a realização do tratamento necessário (TRAVASSOS; PEREIRA; FERNANDES, 2016).

A TARV é indicada para qualquer gestante soropositiva, independente de critérios imunológicos e clínicos. A mesma não deve ser interrompida após parto, devendo ter continuidade do tratamento, independente do nível de LT-CD4+. Àquelas que realizaram o uso correto do TARV, possuir CV-HIV indetectável, deve-se solicitar a próxima contagem na $34^{\mathrm{a}}$ semana gestacional. Há um elevado risco de transmissão vertical, o qual é identificado através da CV-HIV, realização do tratamento durante a gravidez e a relação entre o tempo de uso da TARV corretamente e o parto (BRASIL, 2019).

No município, as gestantes que realizaram o tratamento correto na $\mathrm{AB}$ ou Casa Treze de Maio, podem ter o parto em uma maternidade de risco habitual, como no Hospital Casa de Saúde. Já as que não realizaram as consultas de pré-natal e o tratamento correto, devem ter seu parto em uma maternidade de alto risco, como no HUSM.

Gestantes com HIV não possuem contraindicações para realizar o parto vaginal, porém, são necessários alguns cuidados a serem efetuados, como: ao chegar no serviço iniciar com azidotimidina (AZT) intravenoso até o clampeamento do cordão umbilical; o trabalho de parto deve ser monitorizado criteriosamente, sem realizar toques desnecessários; o clampeamento do cordão umbilical deve ser imediato após a expulsão do feto e não deixar bolsa rota por tempo prolongado (BRASIL, 2019).

Já a cesariana é realizada após a $38^{\mathrm{a}}$ semana de gestação, assim, deve-se certificar a idade gestacional corretamente para evitar prematuridade. Caso a gestante inicie o trabalho de parto antes da cirurgia e apresente dilatação menor que 4 centímetros, deve-se iniciar o AZT, 


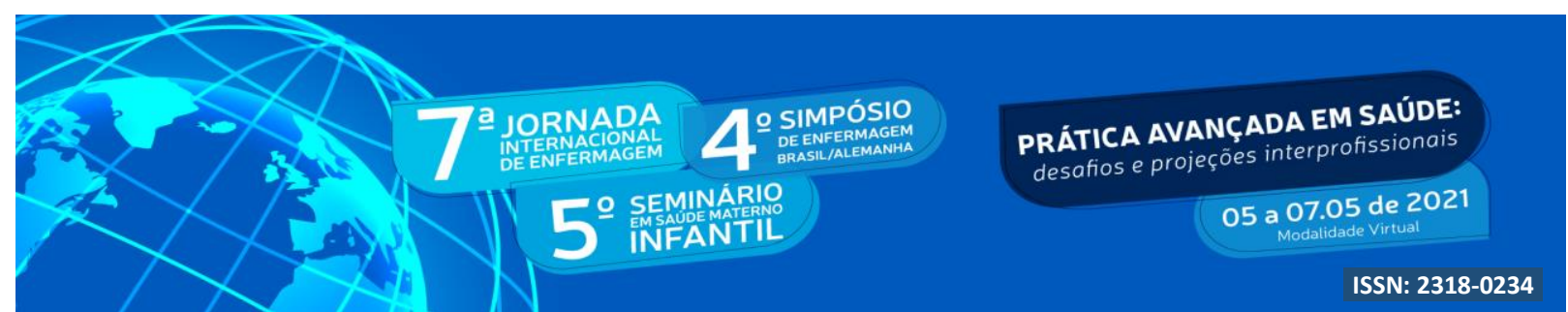

e se possível, realizar a cesárea após 3 horas de infusão, clampeamento de cordão imediato e administrar uma dose de cefazolina durante a cesárea (BRASIL, 2019).

Salienta-se que as gestantes devem ser encaminhadas e/ou transferidas para os serviços de referência de maneira responsável, sendo feito o contato de referência, estabilização do quadro apresentado, relatório clínico e transporte seguro. A figura 1 apresenta o fluxograma desenvolvido pelas acadêmicas de enfermagem sobre o processo de encaminhamento de gestantes com HIV baseado na realidade local do município de Santa Maria/RS.

Figura 1: Processo de encaminhamento de gestantes com HIV baseado na realidade local do município de Santa Maria/RS.

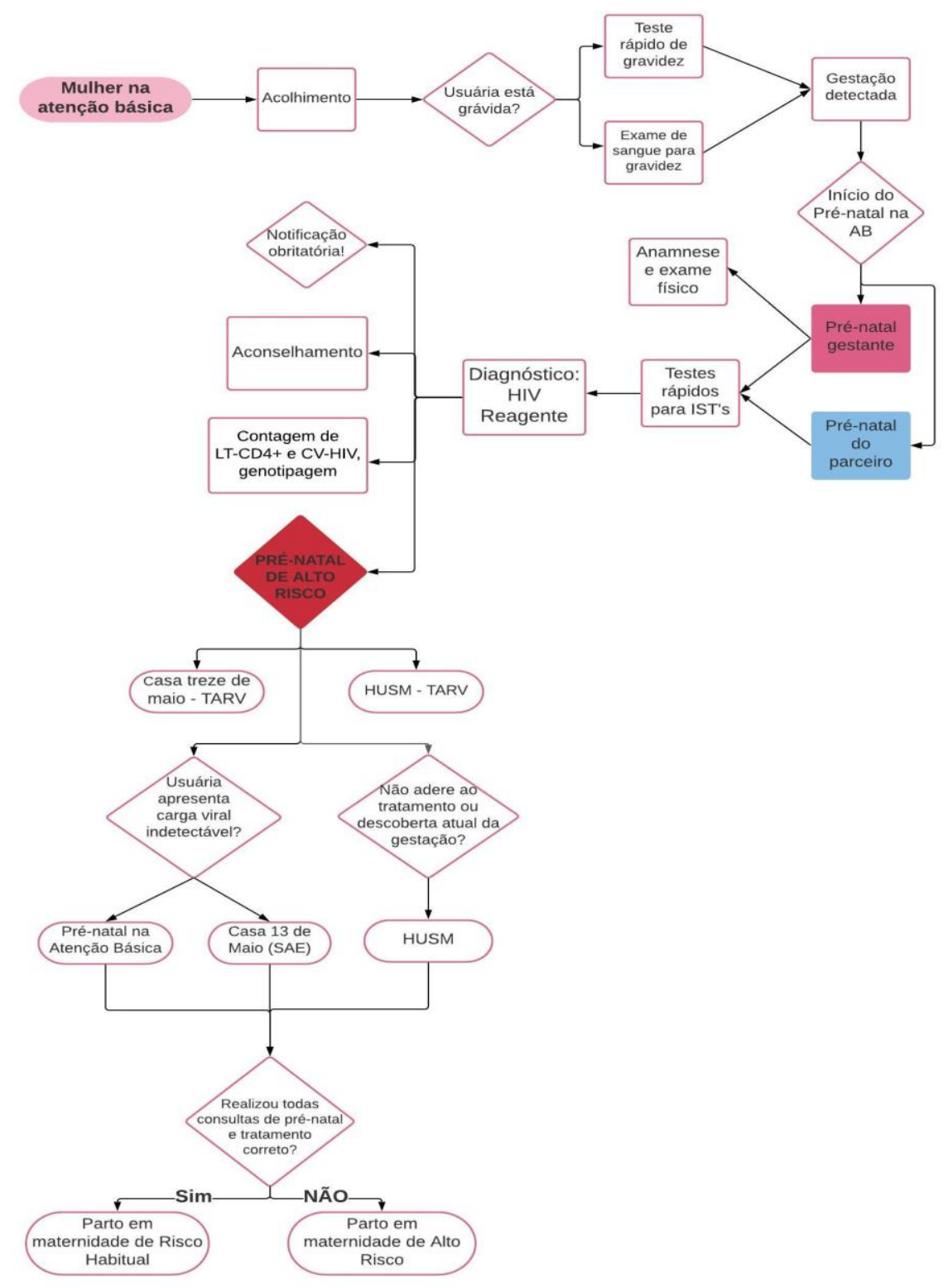




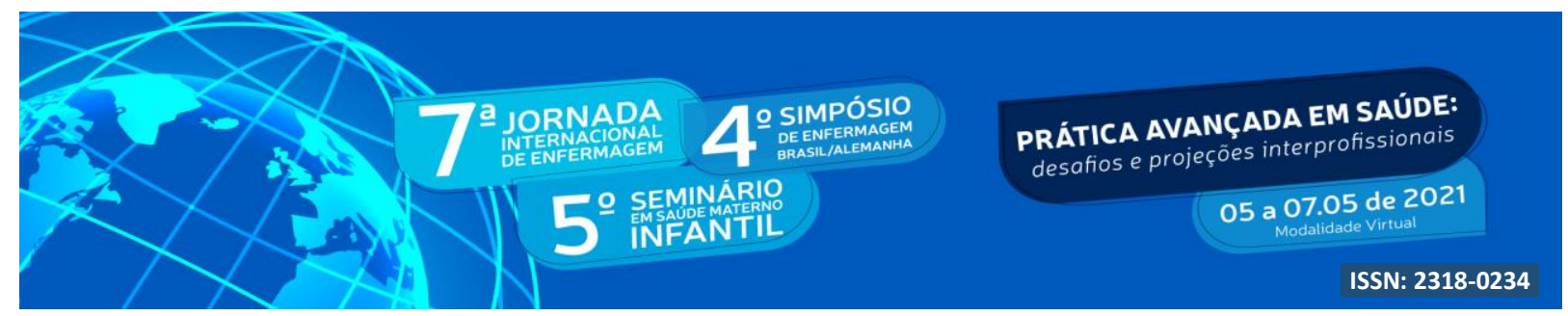

Fonte: desenvolvido pelas autoras.

\section{CONCLUSÃO}

É extremamente importante a realização do pré-natal e a adesão ao tratamento em pacientes obstétricas, pois quando ambos são feitos corretamente e com um acompanhamento profissional, os riscos de complicações e de transmissão vertical diminuem. Para isso, os profissionais da saúde devem adotar algumas medidas básicas sobre o HIV na gestação, como abordá-lo nas consultas de pré-natal, realização em tempo hábil dos exames, notificação compulsória, explicar à gestante sobre a doença e o tratamento e realizar os devidos encaminhamentos. Deve também, estar capacitado para interagir com o público que encontrase nessa situação, buscando compreender o contexto da realidade que estão inseridos, seus hábitos, crenças, atitudes e valores.

Por fim, o profissional enfermeiro tem a capacidade de intervir como um facilitador e estimulador do cuidado, tanto no serviço de saúde quanto a pacientes portadores do vírus HIV. Uma dessas intervenções deve ser no processo de encaminhamento dos usuários nas RAS, tornando de suma importância a elaboração de fluxogramas capazes de contribuir no cuidado continuado e promover a compreensão por parte das gestantes de quais locais devem procurar em diferentes contextos.

\section{REFERÊNCIAS}

BRASIL. Ministério da Saúde. Secretaria de Atenção à Saúde. Departamento de Atenção Básica. Atenção ao pré-natal de baixo risco [recurso eletrônico] / Ministério da Saúde. Secretaria de Atenção à Saúde. Departamento de Atenção Básica. - 1. ed. rev. - Brasília: Editora do Ministério da Saúde, 2013.

BRASIL. Ministério da Saúde. Secretaria de Atenção à Saúde. Departamento de Ações Programáticas Estratégicas. Gestação de alto risco: manual técnico / Ministério da Saúde, Secretaria de Atenção à Saúde, Departamento de Ações Programáticas Estratégicas. - 5. ed. Brasília : Editora do Ministério da Saúde, 2010.

BRASIL. Ministério da Saúde. Secretaria de Vigilância em Saúde. Programa Nacional de DST e Aids. Recomendações para Profilaxia da Transmisão Vertical do HIV e Terapia Antirretroviral em Gestantes: manual de bolso / Ministério da Saúde, Secretaria de 


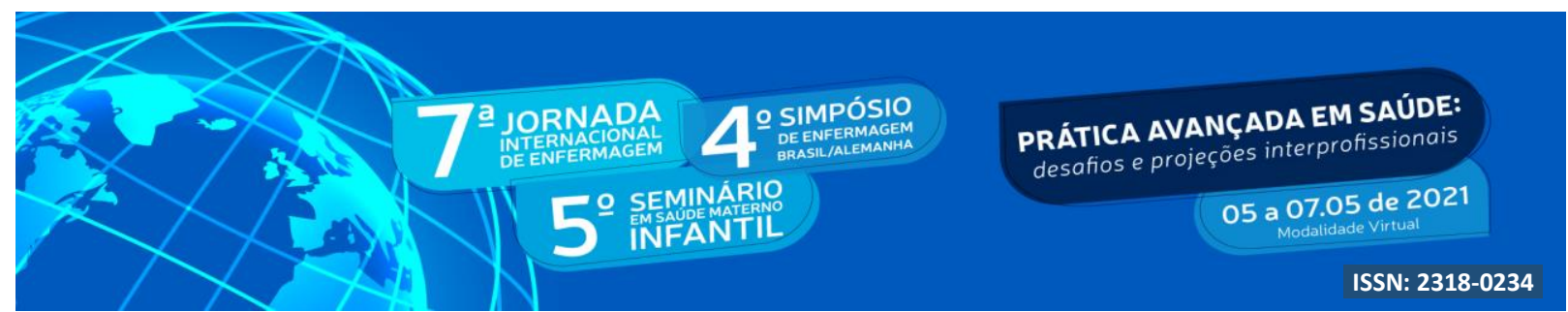

Vigilância em Saúde, Programa Nacional de DST e Aids. - Brasília: Ministério da Saúde, 2010 .

BRASIL. Ministério da Saúde. Biblioteca Virtual em Saúde. HIV e aids. 2017. Disponível em: http://bvsms.saude.gov.br/dicas-em-saude/2409-hiv-e-aids. Acesso em 4 de dez. de 2020.

BRASIL. Ministério da Saúde. Protocolo clínico e diretrizes terapêuticas para prevenção da transmissão vertical de hiv, sífilis e hepatites virais. $1^{a}$ ed. rev. Brasilia-DF. 2019. http://www.aids.gov.br/pt-br/pub/2017/protocolo-clinico-e-diretrizes-terapeuticas-paramanejo-da-infeccao-pelo-hiv-em-criancas-e. Acesso em 5 de dez. de 2020.

BRASIL. Ministério da Saúde. Secretaria de Vigilância em Saúde. Departamento de Vigilância, Prevenção e Controle das Infecções Sexualmente Transmissíveis, do HIV/Aids e das Hepatites Virais. Cuidado integral às pessoas que vivem com HIV pela Atenção Básica: manual para a equipe multiprofissional. Brasília: Ministério da Saúde, 2017. 56 p.

BRASIL. Ministério da Saúde. Secretaria de Vigilância em Saúde. Departamento de Doenças de Condições Crônicas e Infecções Sexualmente Transmissíveis. Protocolo Clínico e Diretrizes Terapêuticas para Prevenção da Transmissão Vertical do HIV, Súfilis e Hepatites Virais, 248 p., 2019.

BRASIL. Ministério da Saúde. Departamento de doenças de Condições Crônicas e Infecções Sexualmente Transmissíveis. Pré Natal, 2020. Disponível em: http://www.aids.gov.br/ptbr/publico-geral/prevencao-combinada/pre-

natal\#: :text=As\%20gestantes\%20que\%20forem\%20diagnosticadas,do\%20HIV\%20para\%20 a\%20crian\%C3\%A7a. Acesso em 10 de mar. de 2020.

BRASIL. Ministério da Saúde. Secretaria de Atenção à Saúde, Núcleo Técnico da Política Nacional de Humanização. Humanização do parto e nascimento [Internet]. Brasília: Ministério da Saúde, 2014. Disponível em: http://www.redehumanizasus.net/sites/defa ult/files/caderno_humanizasus_v4_humanizac ao_parto.pdf

BRASIL. Ministério da Saúde (MS). Política Nacional de Atenção Básica. Brasília: Ministério da Saúde; 2012.

GIL, A. C. Como elaborar projetos de pesquisa. $4^{\text {a }}$ ed. São Paulo: Atlas; 2007.

TRAVASSOS, A. G. A.; PEREIRA, I. N.; FERNANDES, S. A. Assistência à Mulher HIV + no Ciclo Gravídico-Puerperal. EBSERH. Maternidade Climério de Oliveira. Universidade Federal da Bahia. Cap. 10. 2016. Disponivel em: http://www2.ebserh.gov.br/documents/215335/4407336/Protocolo+Infeccoes+na+Gestacao++HIV.pdf/c0d59f4c-deee-4a1f-afe6-735aa792b4f4. Acesso em 3 de dez. de 2020.

VIELAS, E. F. et al. Assistência pré-natal no Brasil. Cad. Saúde Pública, Rio de Janeiro, 30 Sup: S85-S100, 2014. 